\title{
Una estimación indirecta de las desigualdades actuales y futuras en la frecuencia de la enfermedad renal crónica terminal en México
}

\author{
Francisco Franco-Marina, MC, MPH, MHSc, (I) Laura Leticia Tirado-Gómez, MC, MHSc, DSc, ${ }^{(2,3)}$ \\ Aída Venado Estrada, MC, (2) José Andrés Moreno-López, MC, (2) Reyna Lizette Pacheco-Domínguez, MC, MSc, (2) \\ Luis Durán-Arenas, MC, PhD, (2) Malaquías López-Cervantes, MC, MPH, PhD.(2)
}

\begin{abstract}
Franco-Marina F, Tirado-Gómez LL, Venado-Estrada A, Moreno-López JA, Pacheco-Domínguez RL, Durán-Arenas L, López-Cervantes $M$. Una estimación indirecta de las desigualdades actuales y futuras en la frecuencia de la enfermedad renal crónica terminal en México. Salud Publica Mex 20II;53 supl 4:S506-S5I 5.
\end{abstract}

\section{Resumen}

Objetivo. Describir las desigualdades actuales y futuras de la enfermedad renal crónica terminal (ERCT) en México, que se presentan entre grupos de entidades federativas con diferentes grados de marginación. Material y métodos. Partiendo de una estimación indirecta de la incidencia, prevalencia, mortalidad y duración promedio que realizamos en 2009, presentamos datos agrupados de acuerdo con el grado de marginación estatal. Medimos la desigualdad con el Indice de Concentración de Salud. Resultados. Encontramos desigualdades crecientes entre 2005 y 2025 en las tasas de incidencia, prevalencia y mortalidad, así como en la duración promedio de los casos. Conclusión. Para 2025 esperamos importantes incrementos en la prevalencia de la ERCT que afectarán en mayor medida a los estados más marginados, lo que aumentará la inequidad presente en este problema de salud y representará importantes retos para el financiamiento de los servicios de salud, si no se incide sobre las causas y la progresión hacia la ERCT.

Palabras claves: desigualdad en salud; inequidad en salud; incidencia; enfermedad renal crónica terminal; México

\author{
Franco-Marina F, Tirado-Gómez LL, Venado-Estrada A, \\ Moreno-López JA, Pacheco-Domínguez RL, \\ Durán-Arenas L, López-Cervantes M. \\ An indirect estimation of current and future \\ inequalities in the frequency of end stage \\ renal disease in Mexico.
}

Salud Publica Mex 20II;53 suppl 4:S506-S5I 5.

\begin{abstract}
Objective. To describe current and future health inequalities in End Stage Renal Disease in Mexico (ESRD) in Mexican states with varying degrees of marginality. Material and Methods. Using results, obtained by us in 2009 , of an indirect estimation of incidence, prevalence, and mortality rates, and of the average case duration, we grouped these data according to the social deprivation level of the Mexican states. We measured health inequalities using the Health Concentration Index. Results. We found rising inequalities, between 2005 and 2025, in ESRD incidence, prevalence and mortality rates, as well as in the average duration of cases. Conclusion. We project an important increase in the prevalence of ESRD for 2025 which will be greater in the Mexican states with more marginality. This will increase health inequities already present and represent important challenges for health care financing, especially if no action is taken to control the causes and progression of ESRD.
\end{abstract}

Keywords: health inequality; health inequity; incidence; end stage renal disease; Mexico

Una porción de los datos analizados en esta publicación provienen de la Encuesta Nacional de Salud y Nutrición 2006, diseñada y conducida por el Instituto Nacional de Salud Pública

(I) Instituto Nacional de Enfermedades Respiratorias. México.

(2) Facultad de Medicina, UNAM. México.

(3) Instituto Nacional de Pediatría. México

Fecha de recibido: 30 de agosto de 2010 - Fecha de aceptado: I3 de octubre de 201 I

Autor de correspondencia: Dr. Malaquías López-Cervantes. Unidad de Proyectos Especiales de Investigación, Facultad de Medicina, Universidad Nacional Autónoma de México. Torre de Investigación, Ier. piso, Ciudad Universitaria, 045I0 México DF. Correo electrónico: mlopez@liceaga.facmed.unam.mx; mlopezl4@unam.mx 
L a frecuencia de la enfermedad renal crónica muestra una tendencia creciente, tanto en países desarrollados como en aquellos en vías de desarrollo. ${ }^{1-4}$ Una tendencia similar se ha observado para la etapa más avanzada de este padecimiento, la enfermedad renal crónica terminal (ERCT), caracterizada por tasas de filtración glomerular menores a $15 \mathrm{ml} / \mathrm{min} / 1.73$ metros cuadrados. ${ }^{5}$

Se estima que a nivel mundial existen actualmente dos millones de personas con ERCT y que en la presente década se requerirá más de un billón de dólares para su tratamiento. ${ }^{6}$ El elevado costo del tratamiento de la ERCT representará un importante reto para los sistemas de salud, en especial los de los países en desarrollo. ${ }^{7-9}$

Gran parte del incremento observado en la frecuencia de la ERCT se debe a la transición epidemiológica que se experimenta en muchas regiones del mundo, derivada tanto del envejecimiento poblacional como de la adopción de estilos de vida no saludables ${ }^{10}$ que han favorecido el incremento de la obesidad ${ }^{11,12}$ y de padecimientos asociados con la misma, como la diabetes y la hipertensión arterial ${ }^{4,13,14}$ y en los cuales la ERCT es una complicación común si su tratamiento no es adecuado.

En este trabajo se describen las desigualdades actuales y futuras de la ERCT en México que se presentan entre grupos de entidades federativas con diferentes grados de marginación. La desigualdad en salud se manifiesta por la existencia de diferenciales en la frecuencia de los problemas de salud en grupos humanos con diferentes niveles socioeconómicos en los que los grupos menos favorecidos presentan mayor afectación. ${ }^{15}$ Además, cuando las desigualdades en salud son evitables e injustas se está ante inequidades de salud, derivadas de diferenciales inaceptables en términos del financiamiento de los servicios de salud y del acceso a los mismos. ${ }^{16,17}$ En el caso de la ERCT, las desigualdades en salud son inequidades porque se trata de un síndrome originado principalmente por problemas de salud crónicos con deficiente manejo, que con frecuencia afectan más a los grupos con menor acceso a servicios de salud de adecuada calidad y efectividad. ${ }^{18-21}$ Asimismo, la supervivencia de las personas con ERCT es afectada adversamente por la detección tardía del padecimiento, la carencia de nefrólogos y la falta de acceso regular a la terapia de reemplazo, situaciones más comunes en los grupos sociales menos favorecidos. ${ }^{22,23}$

\section{Material y métodos}

\section{Información analizada}

Para este análisis utilizamos información derivada de un ejercicio más detallado ${ }^{24}$ en el que obtuvimos estimaciones indirectas de los siguientes parámetros epide- miológicos de la ERCT en México: tasas de incidencia, prevalencia y mortalidad, así como duración promedio de un caso. Todas las estimaciones se realizaron a nivel estatal, con desagregación por sexo y grupos de edad (0-19, 20-44, 45-64, 65-74 y 75 y más años), de manera quinquenal entre 2005 y 2025. La metodología empleada para obtener estas estimaciones permitió considerar tanto la información disponible en la literatura biomédica, nacional e internacional, como la derivada del análisis de bases de datos de mortalidad y de opiniones de expertos, quienes revisaron las estimaciones en relación con su plausibilidad y aportaron información sobre su experiencia en el manejo de la enfermedad. A continuación presentamos el procedimiento de estimación, el cual se describe con mayor detalle en otra publicación. ${ }^{24}$

\section{Estimaciones para 2005}

La figura 1 resume el proceso de estimación seguido, basado en el empleo de un modelo matemático que permite obtener parámetros epidemiológicos congruentes de determinada enfermedad en una población específica, a partir de la especificación de las tasas de incidencia, letalidad y remisión de la enfermedad. ${ }^{25}$

Asumimos que en México el patrón de afectación por edad y sexo, tanto de las tasas de incidencia como de las de letalidad, debía ser similar al observado en la información del Sistema de Datos Renales de Estados Unidos (USRDS, por sus siglas en inglés), ${ }^{26}$ dado que dicho patrón tiene una determinación fundamentalmente biológica. ${ }^{27}$ Adicionalmente, supusimos que, a nivel nacional, las tasas de letalidad deberían ser, en promedio, el triple de las observadas en el reporte de 2008 del USRDS para la población norteamericana, en consonancia con un estudio realizado de registro de enfermedad renal de Jalisco, único en el país en el que se reportan riesgos de muerte tres veces mayores que los observados en hispanos incluidos en el USRDS. ${ }^{28}$ Asimismo, asumimos que la incidencia de ERCT en México debería ser en promedio de 400 por millón, el doble de la observada en Estados Unidos, dada la menor detección y tratamiento oportuno del daño renal asociado con padecimientos como la diabetes e hipertensión existentes en nuestro país. Por otro lado, debido al limitado número de trasplantes renales que se realizan en México, ${ }^{29}$ se estableció en las estimaciones una tasa de remisión de cero.

El siguiente paso fue obtener estimaciones iniciales de las tasas de incidencia y letalidad a nivel estatal que alimentaran el modelamiento matemático, cumpliendo con las especificaciones nacionales. Para ello se determinó la frecuencia en que la ERCT intervino en las defunciones registradas en México en 2005. Así, anali- 


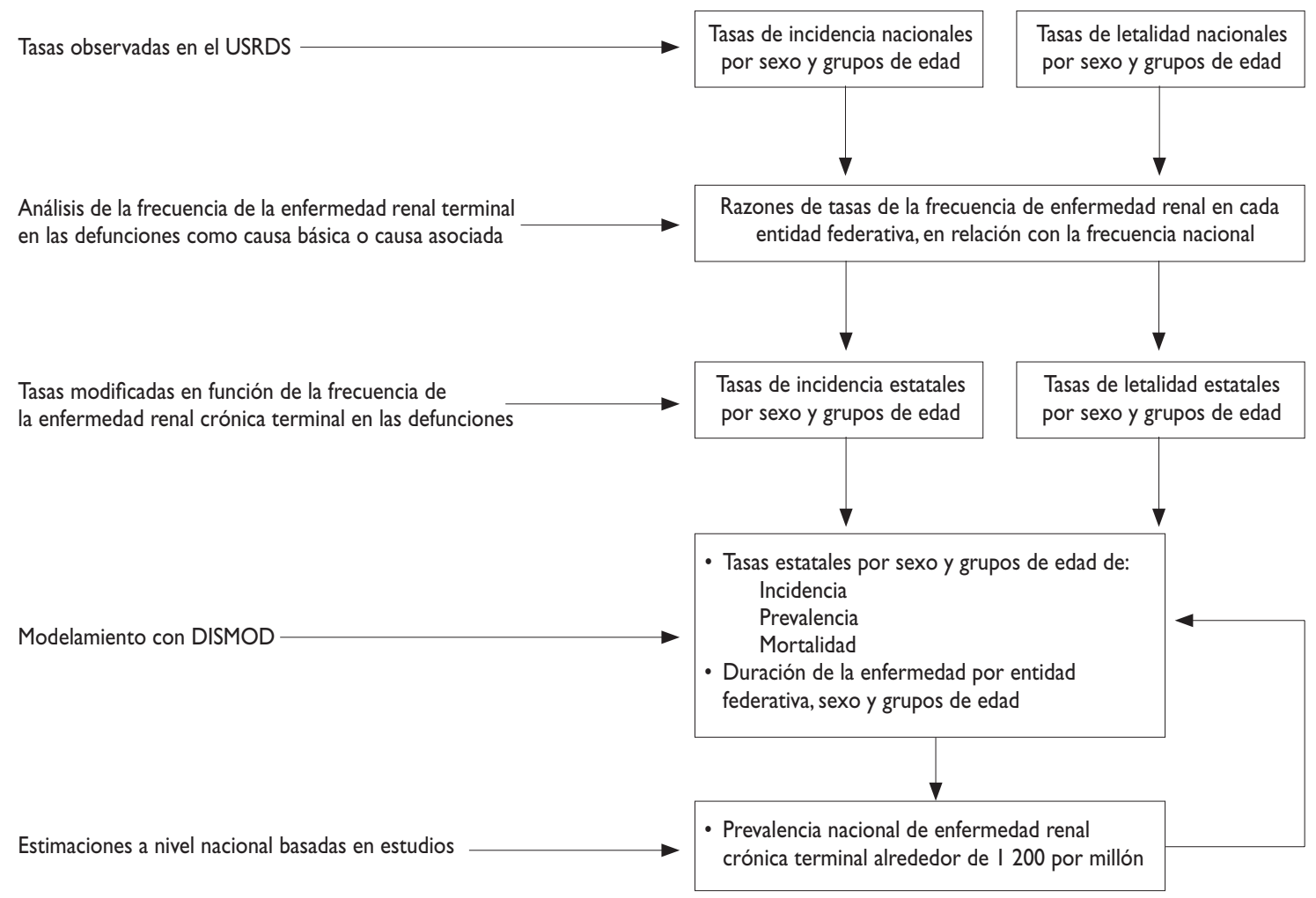

Figura I. Proceso empleado para la estimación de los parámetros epidemiológicos de la enfermedad Renal CRónica TERMINAL PARA 2005

zamos las bases del Sistema Epidemiológico Estadístico de Defunciones (SEED), correspondientes a 2005-2007, compiladas independientemente por la Secretaría de Salud, a partir de las copias de los certificados de defunción remitidos a la misma y que, a diferencia de las bases de mortalidad generadas por INEGI, registran todas las causas de muerte consignadas en el certificado y no sólo la causa básica. Dicho análisis permitió establecer las proporciones de las defunciones atribuidas a 37 causas básicas distintas de la ERCT, en las que existió además mención de ERCT. Al aplicar estas proporciones a las bases de mortalidad generadas por INEGI en 2005, las que por otro lado registran un volumen mayor de defunciones que las bases del SEED, encontramos que en 73799 de las defunciones ocurridas en ese año los fallecidos cursaban con ERCT, aunque en sólo 12162 de las mismas dicha patología fue considerada la causa básica de la defunción. Así se pudieron generar tasas de mortalidad asociadas con la ERCT, como causa básica o contribuyente a la defunción, a nivel estatal y con desagregación por sexo y grupos de edad y, consecuentemente, razones de tasas de mortalidad en relación con el nivel nacional que fueron aplicadas a las tasas de incidencia y letalidad nacionales asumidas para generar las estimaciones estatales iniciales empleadas en el modelo matemático, bajo el supuesto de que las variaciones detectadas en la mortalidad reflejaban también variaciones en la incidencia y en la letalidad.

El modelo matemático empleado es similar a una tabla de vida con decrementos múltiples en la que se hace pasar a una cohorte hipotética, a lo largo de la vida, a través de los estadios de ausencia de la enfermedad, presencia de enfermedad y muerte, utilizando las tasas de incidencia, remisión y letalidad proporcionadas, así como los riesgos de muerte por todas las causas a los que está sometida la población para la que se realiza la estimación. El modelo se encuentra implementado en el programa de cómputo DISMOD II.

Como lo indica la figura 1, el modelamiento matemático consistió en un proceso iterativo en el que las tasas estatales de incidencia y letalidad fueron modificadas sucesivamente, hasta alcanzarse una prevalencia cercana a 1200 por millón, cifra consistente con la única encuesta poblacional en la que se ha realizado una me- 
dición directa de enfermedad renal en México ${ }^{30}$ y con la prevalencia reportada en un análisis del registro de enfermedad renal de Jalisco. ${ }^{26}$

\section{Proyecciones hasta 2025}

Se realizó también un modelamiento matemático para obtener proyecciones estatales de los parámetros epidemiológicos de la ERCT, hasta el año 2025. La proyección realizada se basó en el supuesto de que, hasta 2025, las tasas de letalidad permanecerían en los niveles especificados para el año 2005, toda vez que las terapias dialíticas cubren en la actualidad a una modesta proporción de los casos y tienen un costo relativamente alto para las instituciones de salud. Asimismo, se supuso que entre 2005 y 2025 las tendencias de las tasas de incidencia tendrían, en los diferentes estados, el mismo comportamiento que el proyectado para las tasas de mortalidad asociadas con la ERCT.

Para realizar las proyecciones hasta 2025 en las tasas de mortalidad asociadas con la ERCT, primeramente se aplicaron las proporciones de defunciones atribuidas a 37 causas básicas de defunción en las que coexistiría la enfermedad renal crónica, obtenidas previamente, a la mortalidad registrada entre 1990 y 2004 para obtener el volumen de defunciones en personas que cursaron con ERCT. Enseguida, se ajustó con esta serie, en cada sexo, grupo de edad y estado, modelos de regresión lineal de Poisson, con los cuales se obtuvieron predicciones de las tasas de mortalidad entre 2010 y 2025 y a partir de ellas se hicieron variar proporcionalmente las tasas de incidencia.

\section{Análisis estadístico}

Se colapsó la información sobre los parámetros epidemiológicos que se generaron previamente a nivel estatal en cinco estratos de marginación, basados en el grado de marginación de las entidades federativas generado por el Consejo Nacional de Población (CONAPO) para 2005. ${ }^{31}$

Se analizó esta información para tres grupos de edad (0-19, 20-44 y 45 más años), en vez de los cinco originalmente utilizados en el ejercicio de estimación, porque los hallazgos en los grupos de edad de 45 años y mayores fueron muy similares. Tampoco se desagregó la información por sexo pues no hubo diferencias importantes en las estimaciones para hombres y mujeres.

Se realizaron además dos análisis de las bases de datos de la Encuesta Nacional de Salud y Nutrición (ENSANUT 2006), ${ }^{32}$ empleando modelos de regresión logística en los que se consideraron los ponderadores individuales existentes en dichas bases. El primer análisis se hizo para determinar el porcentaje de la población sin acceso a seguro médico privado o proporcionado por alguna institución de seguridad social en los cinco estratos de marginación. El segundo análisis consistió en la estimación de la prevalencia relativa, ajustada por edad y sexo, de diabetes e hipertensión, definidas como indicamos al pie del cuadro I, para los cinco estratos de marginación.

Finalmente, se empleó como medida de desigualdad al Índice de Concentración de Salud (ICS), análogo del índice GINI, utilizado en economía para medir la concentración del ingreso y que permite medir la desigualdad en términos de eventos de salud. O'Donnell y cols. ${ }^{33}$ definen el ICS como el doble del área en una curva de desigualdad de Lorenz entre la curva de concentración de las tasas y la línea de igualdad, y proporcionan fórmulas para su cálculo y para la obtención de su error estándar.Aquí el ICS se expresó como porcentaje. En este trabajo debe interpretarse que un valor negativo del ICS indica que los parámetros epidemiológicos contrastados son menos favorables en los grupos más pobres o marginados, mientras que un valor positivo indicaría una situación más favorable en dichos grupos.

\section{Resultados}

El cuadro I presenta información relevante sobre las características demográficas y de necesidades de salud de los cinco grupos de estados, formados según su grado de marginación en 2005. Mientras que en 2005 50.7\% de la población mexicana residía en estados con baja o muy baja marginación, 35.6\% lo hacía en aquéllos con alta o muy alta marginación. En 2005, la población de los estados con muy alta marginación tenía una edad promedio 3.4 años menor que el promedio de las entidades de más baja marginación. En cambio, para 2025 esta diferencia disminuirá a 2.8 años, lo que implica un proceso de envejecimiento poblacional más acelerado en los estados más marginados.

Por otro lado, la información de ENSANUT 2006 muestra que el acceso a servicios de salud proporcionados por la seguridad social o por seguros médicos privados aumenta gradualmente a medida que disminuye el grado de marginación, desde $47.2 \%$ de la población con acceso a estos servicios en las entidades más marginadas hasta $76.1 \%$ de la población que residía en los estados con menor marginación. Además, las tasas de prevalencia de diabetes e hipertensión arterial, ajustadas por edad y sexo, son considerablemente menores en las entidades de muy alta marginación que las observadas en las de muy baja marginación (razones de prevalencia de 0.57 y 0.69 , respectivamente).

La figura 2 presenta la evolución de la mortalidad asociada con ERCT como causa básica o contribuyente a 


\section{Cuadro I}

Características demográficas y necesidades de salud de los grupos de entidades federativas de México, AGRUPADAS SEGÚN SU GRADO DE MARGINACIÓN EN 2005

\begin{tabular}{|c|c|c|c|c|c|c|c|}
\hline \multirow{3}{*}{$\begin{array}{l}\text { Grado de } \\
\text { marginación* }\end{array}$} & \multirow[b]{3}{*}{ Entidades federativas incluidas } & \multirow{3}{*}{$\begin{array}{l}\text { oblación en } 2005^{\ddagger} \\
\quad \text { (en miles) }\end{array}$} & \multirow{2}{*}{\multicolumn{2}{|c|}{$\begin{array}{c}\text { Edad promedio de } \\
\text { la población } n^{\ddagger}\end{array}$}} & \multirow{3}{*}{$\begin{array}{c}\text { \% de la población } \\
\text { sin seguridad socialß }\end{array}$} & \multicolumn{2}{|c|}{ Razón de prevalencias $\#$ para: } \\
\hline & & & & & & \multirow[b]{2}{*}{ Diabetes } & \multirow{2}{*}{$\begin{array}{l}\text { Hipertensión } \\
\text { arterial }\end{array}$} \\
\hline & & & 2005 & 2025 & & & \\
\hline luy alta & Chiapas, Guerrero, Oaxaca & $11,020.3$ & 25.9 & 33.0 & $\begin{array}{c}76.1 \\
(72.8-79.2)\end{array}$ & $\begin{array}{c}0.57 \\
(0.44-0.74)\end{array}$ & $\begin{array}{c}0.69 \\
(0.59-0.80)\end{array}$ \\
\hline Ita & $\begin{array}{l}\text { Campeche, Hidalgo, Michoacán, Puebla, San Luis } \\
\text { Potosí, Tabasco, Veracruz, Yucatán }\end{array}$ & $26,035.0$ & 27.4 & 34.5 & $\begin{array}{c}73.1 \\
(70.7-75.4)\end{array}$ & $\begin{array}{c}0.58 \\
(0.46-0.72)\end{array}$ & $\begin{array}{c}0.97 \\
(0.86-1.10)\end{array}$ \\
\hline edia & $\begin{array}{l}\text { Durango, Guanajuato, Nayarit, Querétaro, Sinaloa, } \\
\text { Tlaxcala, Zacatecas }\end{array}$ & $|4||| .2$, & 27.0 & 34.4 & $\begin{array}{c}65.6 \\
(63.1-68.0)\end{array}$ & $\begin{array}{c}0.77 \\
(0.62-0.97)\end{array}$ & $\begin{array}{c}1.13 \\
(1.01-1.27)\end{array}$ \\
\hline Baja & $\begin{array}{l}\text { Aguascalientes, Baja California Sur, Chihuahua, } \\
\text { Colima, Jalisco, México, Morelos, Quintana Roo, } \\
\text { Sonora, Tamaulipas }\end{array}$ & $34,405.2$ & 27.4 & 34.3 & $\begin{array}{c}59.4 \\
(56.8-61.8)\end{array}$ & $\begin{array}{c}0.79 \\
(0.63-0.98)\end{array}$ & $\begin{array}{c}1.03 \\
(0.91-1.17)\end{array}$ \\
\hline Muy baja & $\begin{array}{l}\text { Baja California, Coahuila, } \\
\text { Distrito Federal, Nuevo León }\end{array}$ & $18,375.2$ & 29.3 & 35.8 & $\begin{array}{c}47.2 \\
(44.4-50.0)\end{array}$ & I & 1 \\
\hline \multicolumn{8}{|c|}{$\begin{array}{l}\text { \# Razones de prevalencias ajustadas por edad y sexo en un modelo de regresión logística con datos ponderados, ajustado en personas de } 18 \text { años o más por } \\
\text { los autores de este trabajo, empleando las bases de la ENSANUT } 2006^{32} \text { Las razones de prevalencia utilizan como referente el grupo de entidades con muy } \\
\text { baja marginación. La presencia de diabetes se estableció mediante la respuesta afirmativa por parte de los entrevistados a la pregunta sobre diagnóstico previo } \\
\text { realizado por un médico o si el resultado de la glucemia en ayunas era mayor a I } 25 \mathrm{mg} \text {-dl o la glucemia casual fue superior o igual a } 200 \mathrm{mg} / \mathrm{dl} \text {. La presencia } \\
\text { de hipertensión arterial se estableció mediante la respuesta afirmativa por parte de los entrevistados a la pregunta sobre diagnóstico previo realizado por } \\
\text { un médico, o si el promedio de las dos mediciones de la tensión arterial realizada en la encuesta fue igual o superior a } 140 \mathrm{mmHg} \text { en el caso de la tensión } \\
\text { sistólica, o igual o superior a } 90 \mathrm{mmHg} \text { en el caso de tensión diastólica }\end{array}$} \\
\hline
\end{tabular}

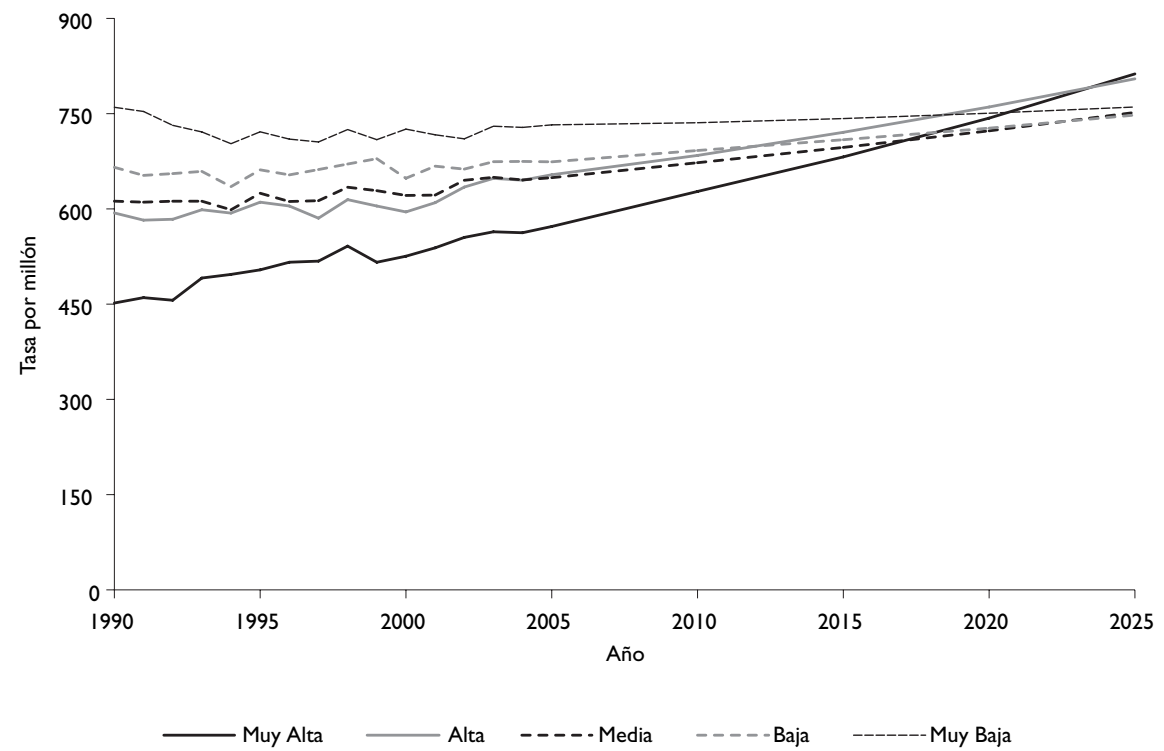

Figura 2. TASAS de MORTALIdAd ASOCIADAS CON ENFERMEDAD RENAL CRÓNICA TERMINAL, OBSERVADAS ENTRE I990 Y 2005 Y PROYECTADAS DESDE ESTE ÚLTIMO AÑO HASTA 2025, PARA LOS CINCO ESTRATOS DE ENTIDADES FEDERATIVAS DE MÉXICO, FORMADOS EN FUNCIÓN DE SU GRADO DE MARGINACIÓN. LAS TASAS PRESENTADAS ESTÁN AJUSTADAS POR EDAD Y SEXO A LA DISTRIBUCIÓN QUE LA POBLACIÓN MEXICANA PRESENTABA EN ESTAS VARIABLES EN 2005, EMPLEANDO EL MÉTODO DIRECTO 
la defunción, observada entre 1990 y 2005 y proyectada hasta el 2025 para los cinco estratos de marginación analizados. Mientras que en 1990 existía un gradiente de menor mortalidad a medida que aumentaba el grado de marginación de los estados, las entidades con alta y muy alta marginación presentaron entre 1990 y 2005 una tendencia al incremento más pronunciada que el observado en el resto de los estados. Consecuentemente, se proyectó que para 2025 las tasas de mortalidad asociadas con ERCT tenderán a ser más similares en los cinco estratos de marginación y que incluso los estados con alta o muy alta marginación tendrán en este año tasas entre 5 y $6 \%$ mayores que las observadas en los estados con menor marginación.

El cuadro II presenta las estimaciones para 2005 de los parámetros epidemiológicos de la ERCT para los cinco estratos de marginación, con desagregación por grupos de edad. Se presentan también los porcentajes de cambio en estos indicadores proyectados para 2025. Al considerar todas las edades, las tasas de incidencia y las de mortalidad debidas a ERCT varían en 2005 entre 312 y 433 por millón en todos los estratos de marginación, proyectándose además para 2025 incrementos en dichas tasas que serán mayores en los estados más

Cuadro II

TASAS DE INCIDENCIA, MORTALIDAD, PREVALENCIA Y DURACIÓN PROMEDIO DE LOS CASOS DE ENFERMEDAD RENAL CRÓNICA TERMINAL POR GRUPOS DE EDAD ESTIMADOS PARA 2005 Y PORCENTAJE DE CAMBIO PROYECTADO PARA 2025 EN CINCO ESTRATOS DE LAS ENTIDADES FEDERATIVAS DE MÉXICO, FORMAdOS EN FUNCIÓN DE SU GRAdo de MARGINACIÓN

\begin{tabular}{|c|c|c|c|c|c|c|c|c|}
\hline \multirow[b]{2}{*}{$\begin{array}{l}\text { Grado de } \\
\text { marginación }\end{array}$} & \multicolumn{2}{|r|}{$\begin{array}{c}\text { Tasa de } \\
\text { incidencia* }\end{array}$} & \multicolumn{2}{|r|}{$\begin{array}{l}\text { Tasa de mortalidad } \\
\text { debida a ERCT* }\end{array}$} & \multicolumn{2}{|r|}{$\begin{array}{c}\text { Tasa de } \\
\text { prevalencia* }\end{array}$} & \multicolumn{2}{|r|}{$\begin{array}{l}\text { Duración promedio de } \\
\text { un caso, en años }\end{array}$} \\
\hline & 2005 & $\begin{array}{l}\text { \% de cambio proyectado } \\
\text { entre } 2005 \text { y } 2025\end{array}$ & 2005 & $\begin{array}{l}\text { \% de cambio proyectado } \\
\text { entre } 2005 \text { y } 2025\end{array}$ & 2005 & $\begin{array}{l}\text { \% de cambio proyectado } \\
\text { entre } 2005 \text { y } 2025\end{array}$ & 2005 & $\begin{array}{l}\text { \% de cambio proyectado } \\
\text { entre } 2005 \text { y } 2025\end{array}$ \\
\hline \multicolumn{9}{|c|}{ Todas las edades } \\
\hline Muy alta & 433 & 133.7 & 407 & 141.6 & 1209 & 83.0 & 4.5 & -32.9 \\
\hline Alta & 402 & 92.2 & 374 & 99.4 & 1242 & 56.6 & 4.7 & -27.0 \\
\hline Media & 385 & 90.0 & 356 & 97.1 & 1196 & 53.2 & 4.8 & -28.7 \\
\hline Baja & 388 & 63.9 & 355 & 71.1 & $|29|$ & 34.4 & 4.9 & -23.5 \\
\hline Muy baja & 341 & 53.0 & 312 & 60.1 & 1216 & 26.2 & 5.2 & -22.2 \\
\hline
\end{tabular}

$0-19$ años

\begin{tabular}{lllllllll} 
Muy alta & 39 & -49.1 & 15 & -49.4 & 291 & -49.1 & 11.2 & -0.8 \\
\hline Alta & 29 & -47.3 & II & -44.3 & 225 & -47.2 & 12.0 & 0.0 \\
\hline Media & 30 & -46.6 & II & -46.2 & 229 & -45.7 & 12.2 & -0.1 \\
\hline Baja & 30 & -39.5 & 11 & -36.8 & 235 & -38.0 & 12.2 & -0.6 \\
\hline Muy baja & 24 & -44.7 & 9 & -41.5 & 192 & -43.3 & 12.7 & -0.9 \\
\hline
\end{tabular}

20-44 años

\begin{tabular}{llllrrrrr} 
Muy alta & 152 & -15.6 & 135 & -13.6 & 977 & -17.2 & 6.3 & -3.2 \\
\hline Alta & 165 & -13.9 & 136 & -11.7 & 1087 & -14.1 & 6.8 & -2.6 \\
\hline Media & 144 & -23.3 & 120 & -20.6 & 983 & -23.2 & 7.0 & -3.1 \\
\hline Baja & 169 & -10.9 & 139 & -9.7 & 1146 & -11.9 & 6.9 & -2.4 \\
\hline Muy baja & 143 & -6.5 & 113 & -5.3 & 1039 & -9.0 & 7.5 & -3.4 \\
\hline
\end{tabular}

45 y más años

\begin{tabular}{lllllllll} 
Muy alta & 1983 & 60.9 & 1931 & 61.6 & 4007 & 54.4 & 2.4 & -3.7 \\
\hline Alta & 1636 & 31.1 & 1587 & 31.9 & 3697 & 26.9 & 2.6 & -3.2 \\
\hline Media & 1662 & 24.2 & 1601 & 25.0 & 3799 & 20.2 & 2.7 & -3.4 \\
\hline Baja & 1614 & 8.4 & 1547 & 10.1 & 3848 & 3.7 & 2.8 & -4.5 \\
\hline Muy baja & 1254 & 4.9 & 1202 & 6.8 & 3257 & -1.0 & 3.0 & -6.1
\end{tabular}

* por millón de habitantes 
marginados. La prevalencia estimada para 2005 es cercana a 1200 por millón en todas las edades y muestra sólo ligeras variaciones entre los estratos comparados. No obstante, se proyectó que la prevalencia aumentará entre $26.2 \%$ en los estados con muy baja marginación y $83 \%$ en los estados con muy alta marginación. Por otro lado, la duración promedio de un caso de ERCT en todas las edades fluctuaba en 2005 entre 4.5 y 5.2 años, y era mayor en los estados con muy baja marginación. Para 2025 se proyectó que, como consecuencia del envejecimiento poblacional, la duración promedio disminuirá en todos los estratos de marginación, aunque en forma más intensa en las entidades con muy alta marginación.

La desagregación de la información por grupos de edad muestra que tanto para la incidencia de la ERCT como para la mortalidad y la prevalencia se anticipa para 2025 una reducción en las respectivas tasas en los menores de 45 años que será más intensa en los estados con muy alta y alta marginación. En cambio, para la población con edades de 45 o más años se proyectan importantes aumentos en las tasas que nuevamente serán más intensos en los estados con muy alta y alta marginación. Por otro lado, la duración promedio de los casos de ERCT sufrirá variaciones marginales durante el periodo analizado en los grupos de edad y estratos de marginación considerados.

Por último, el cuadro III presenta los índices de concentración de los parámetros presentados en el cuadro II. Para todas las edades, en el año 2005 se observan ICS indicativos de una situación menos favorable a medida que aumenta el grado de marginación de los estados, en el caso de las tasas de incidencia y mortalidad (ICS de $-3.3 \%$ y $-3.8 \%$, respectivamente). Además, se proyecta que para 2025 los ICS aumentarán a valores cercanos a $-10.0 \%$ en estos dos parámetros. En cuanto a las tasas de prevalencia se tiene que mientras en 2005 éstas eran muy similares entre los diferentes estratos de marginación, para 2025 anticipamos que serán considerablemente mayores en los estados con alta o muy alta marginación, con un ICS de $-5.7 \%$ en este año. Asimismo, se proyecta una mayor desigualdad en la duración promedio de un caso de ERCT entre 2005 y 2025 (ICS de $-2.4 \%$ y $-4.6 \%$, respectivamente), con menores duraciones a medida que aumenta el grado de marginación.

Las tendencias en la desigualdad en los diferentes parámetros epidemiológicos para todas las edades se replican para el grupo de edad de 45 años y más, en el que incluso anticipamos mayores incrementos en la desigualdad para 2025 que los proyectados para todas las edades. En contraste, en los menores de 45 años se proyectan para 2025 reducciones en la desigualdad existente en varios parámetros en 2005.

\section{Discusión}

Presentamos aquí un análisis de las desigualdades presentes y futuras de la ERCT en México a nivel agregado. La información base de dicho análisis proviene de un modelamiento matemático, a nivel estatal, que permitió obtener estimaciones indirectas pero congruentes de varios parámetros epidemiológicos de la ERCT para los estados de México.

Dada la naturaleza de la estimación, que en modo alguno podría sustituir a la información más precisa que podría obtenerse de un registro nacional de enfermedad renal en México, se discutieron enseguida los resultados obtenidos a la luz de una evaluación de las limitaciones de la información y los supuestos empleados.

Para el año 2005, se encontró, tanto a nivel general como en todos los grupos de edad, mayores tasas de incidencia y mortalidad por ERCT a medida que aumenta el grado de marginación de los estados. Un supuesto de la estimación que puede influir en estos hallazgos es que, ante la carencia de otras fuentes de información, se em-

Cuadro III

ÍNDICES DE CONCENTRACIÓN DE SALUD PARA LOS DIFERENTES PARÁMETROS EPIDEMIOLÓGICOS DE LA ENFERMEDAD RENAL CRÓNICA TERMINAL POR GRUPOS DE EDAD ESTIMADOS PARA 2005 y 2025. MÉXICO

\begin{tabular}{|c|c|c|c|c|c|c|c|c|}
\hline \multirow[b]{2}{*}{ Grupo de edad } & \multicolumn{2}{|c|}{$\begin{array}{l}\text { Tasas de } \\
\text { incidencia }\end{array}$} & \multicolumn{2}{|c|}{$\begin{array}{l}\text { Tasas de } \\
\text { mortalidad }\end{array}$} & \multicolumn{2}{|c|}{$\begin{array}{c}\text { Tasas de } \\
\text { prevalencia }\end{array}$} & \multicolumn{2}{|c|}{$\begin{array}{l}\text { Duración promedio } \\
\text { de un caso, en años }\end{array}$} \\
\hline & 2005 & 2025 & 2005 & 2025 & 2005 & 2025 & 2005 & 2025 \\
\hline $0-19$ años & -4.9 & -2.1 & -6.7 & -3.1 & -4.4 & -1.1 & $-1.7^{\dagger}$ & -1.5 \\
\hline 20-44 años & -0.8 & 1.3 & -2.1 & -0.3 & 1.0 & 2.7 & $-2.3^{\dagger}$ & $-2.2^{\dagger}$ \\
\hline 45 y más años & -5.9 & $-12.9 \dagger$ & -6.2 & $-12.9 \dagger$ & -2.3 & $-9.6 \dagger$ & $-3.4^{\dagger}$ & $-2.9^{\dagger}$ \\
\hline Todas las edades & $-3.3^{\dagger}$ & $-10.2^{\dagger}$ & $-3.8^{\dagger}$ & $-10.5^{\dagger}$ & 0.4 & $-5.7^{\dagger}$ & $-2.4^{\dagger}$ & $-4.6^{\dagger}$ \\
\hline
\end{tabular}

$\dagger p<0.05$ 
plearon las variaciones en las tasas mortalidad asociada con ERCT como base para obtener los niveles estatales de las tasas de incidencia y letalidad empleadas en los modelamientos estatales. No se cuenta con información que permita verificar este supuesto directamente ni se puede descartar que, a pesar de haber empleado un alto volumen de defunciones asociadas con ERCT (casi 74 000), haya existido un subregistro diferencial en la mortalidad asociada con ERCT que hubiera afectado más a los estados más pobres, dado que para la reconstrucción de las muertes asociadas se dependía de la calidad del llenado de los certificados de defunción. No obstante, si éste hubiera sido el caso deberían esperarse mayores tasas de incidencia y letalidad en los estados más pobres del país y consecuentemente mayor desigualdad en la incidencia y mortalidad debida a ERCT que la encontrada.

Por otro lado, la desigualdad en las tasas de incidencia y letalidad identificada está en consonancia con varios hechos. Primeramente, la incidencia de la ERCT en adultos depende tanto de un adecuado manejo de condiciones como la diabetes, ${ }^{34-37}$ la hipertensión arterial $^{38}$ y el síndrome metabólico, como de la detección y tratamiento adecuados de la nefropatía asociada con dichos padecimientos. ${ }^{10}$ Además, la incidencia en niños y jóvenes está asociada principalmente con las anomalías congénitas del riñón y el tracto urinario, así como con la glomerulonefritis post-estreptocócica. ${ }^{39}$ La mayoría de las patologías asociadas con la ERCT dependen entonces de un acceso a servicios de salud efectivo para su control o tratamiento, el cual, según los datos de acceso a seguridad social o seguro médico privado que se presentan, no estaba disponible para la mayor parte de la población de los estados con mayor marginación del país en 2005. Aunado a lo anterior, las tasas de letalidad en personas con ERCT dependen de su acceso a terapias de reemplazo renal, ${ }^{40}$ que son menos frecuentes a medida que aumenta el grado de marginación.

En cuanto a la prevalencia de la ERCT para 2005, encontramos tasas entre 1200 y 1300 por millón, ligeramente mayores que las reportadas en otros países latinoamericanos, ${ }^{41,42}$ pero muy similares entre los estratos de marginación comparados. En cambio, para ese mismo año se detectaron mayores tasas de prevalencia a medida que aumenta el grado de marginación en los grupos de edad de 0 a 20 y de 45 y más años, pero no en el grupo de 20 a 44 años. Lo anterior implica que las tasas de prevalencia para todas las edades reflejan principalmente las encontradas en este último grupo de edad, debido a que es el más numeroso en la población.

Sin desagregación por edad, la presente proyección de la situación epidemiológica de la ERCT para 2025, en relación con 2005, refleja, por un lado, un aumento de todos los parámetros epidemiológicos y, por el otro, una mayor desigualdad. Estas proyecciones dependen de la validez de los dos supuestos en que están sustentadas: que las tasas de letalidad permanecerán constantes entre 2005 y 2025 y que las tasas de incidencia se comportarán como se proyectó que evolucionaría la mortalidad asociada con ERCT. El primer supuesto implica que la fracción de la población que tiene acceso a terapias renales de reemplazo permanecerá más o menos constante en el periodo proyectado, como puede ocurrir dado el elevado costo de tales tratamientos. El segundo supuesto está sustentado en la evolución observada de la mortalidad asociada con ERCT entre 1991 y 2005, con reducciones a futuro en los grupos de edad más jóvenes e incrementos en las edades de 45 años y más. Estas tendencias difícilmente podrían ser atribuibles a cambios en las tasas de letalidad derivados de cambios en el acceso de la población a tratamiento y sí en cambio a las epidemias de enfermedades crónicas asociadas con la ERCT y con la disminución de la glomerulonefritis post-estreptocócica como causa de ERCT en los niños y jóvenes. Además, las tasas de mortalidad de los estados con alta y muy alta marginación experimentarán mayores incrementos en la mortalidad asociada, lo que sugiere que en ellos habrá una intensificación de las epidemias de diabetes e hipertensión.

La medida epidemiológica de mayor interés en términos de la generación de políticas para control de la ERCT mediante terapias sustitutivas es la prevalencia, ya que corresponde al volumen de personas que en cualquier momento requerirán dichas terapias. El hecho de que aquí se anticipen importantes incrementos en la prevalencia en todos los estratos de marginación, pero principalmente en los estratos con mayor marginación, implicará importantes retos a la equidad en salud en el plazo mediano para el país. Así por ejemplo, se proyecta que los casos prevalentes de ERCT que residen en entidades de alta o muy alta marginación pasarán de cerca de 45700 en 2005 a cerca de 80000 en 2025. Más aún, incluso permaneciendo constantes las tasas de incidencia se proyecta que los casos para 2025 en estas entidades serían cercanos a 68 200, simplemente por el envejecimiento poblacional.

El costo de cualquiera de las modalidades de terapia de reemplazo para la ERCT es en general elevado y representa un reto económico para las instituciones de salud públicas en muchos países. ${ }^{5,7,939,43,44}$ Por ello, los mayores incrementos que se proyectaron para las poblaciones más marginadas de México representarán una pesada carga para las instituciones encargadas de prestar servicios de salud a las mismas, como el Seguro Popular, en particular si se opta por proporcionar trata- 
miento de reemplazo para la ERCT en vez de prevenir su aparición desde ahora, por medio de un mejor control de las patologías crónicas asociadas con la misma.

A nivel mundial ha surgido recientemente un interés por detectar y tratar adecuadamente la enfermedad renal crónica para reducir su progresión hacia ERCT. ${ }^{8,39,45-47}$ Incluso, se ha propuesto la realización en pacientes diabéticos e hipertensos atendidos en el primer nivel de atención de exámenes de orina para detectar proteinuria y la determinación de creatinina sérica para estimar la tasa de filtración glomerular. ${ }^{48}$ Existen además intervenciones efectivas y de bajo costo para el tratamiento inicial de la enfermedad renal crónica que incluyen cambios en el estilo de vida y el empleo de medicamentos nefroprotectores. ${ }^{2,3,49}$

De hecho, si se mantuviera en México un acceso a terapias de reemplazo similar a los niveles actuales y se redujeran para 2025 las tasas de incidencia a través de las citadas intervenciones a la mitad de los niveles actuales, se tendría en ese año, en las entidades con alta o muy alta marginación, alrededor de 37400 casos prevalentes en vez de los 45700 que se proyectaron.

En conclusión la presente estimación indirecta indica que en el mediano plazo se esperan importantes incrementos en la prevalencia de la ERCT que afectarán más a los estados más marginados, lo que aumentará la inequidad presente en este problema de salud y representará importantes retos para el financiamiento de los servicios de salud. Por ello es importante la generación de políticas de salud que incidan sobre las causas y la progresión hacia la ERCT.

Declaración de conflicto de intereses: Los autores declararon no tener conflicto de intereses.

\section{Agradecimientos}

Los autores desean agradecer a los doctores Ricardo Correa-Rotter, Juan Alfredo Tamayo y Orozco, Alejandro Treviño Becerra, Ramón Paniagua Sierra, Gregorio Tomás Obrador, Víctor Argueta Villamar, y Guillermo García García, por sus valiosas aportaciones acerca de los supuestos empleados en la estimación indirecta que presentamos aquí.

\section{Referencias}

I. Zhang L, Wang H. Chronic kidney disease epidemic: cost and health care implications in China. Semin Nephrol 2009;29(5):483-486.

2. Glassock RJ. The rising tide of end-stage renal disease: what can be done? Clin Exp Nephrol 2004;8(4):29I-296.

3. Eknoyan G, Lameire N, Barsoum R, Eckardt KU, Levin A, Levin N, et al. The burden of kidney disease: improving global outcomes. Kidney Int 2004;66(4):1310-13|4.
4. Alebiosu CO, Ayodele OE. The global burden of chronic kidney disease and the way forward. Ethn Dis 2005;15(3):418-423.

5. Ploth DW, Shepp PH, Counts C, Hutchison F. Prospective analysis of global costs for maintenance of patients with ESRD. Am J Kidney Dis 2003;42(I):|2-21

6. Klarenbach S, Manns B. Economic evaluation of dialysis therapies. Semin Nephrol 2009;29(5):524-532.

7. Benain JP, Faller B, Briat C, jacquelinet C, Brami M, Aoustin M, et al. Coût de la prise en charge de la dialyse en France. Nephrol Ther 2007;3(3):96-106. 8. Meguid El Nahas A, Bello AK. Chronic kidney disease: the global challenge. Lancet 2005;365(9456):33I-340.

9. Klarenbach S, Barnieh L, Gill J. Is living kidney donation the answer to the economic problem of end-stage renal disease? Semin Nephrol 2009;29(5):533-538

10. Iseki K. Metabolic syndrome and chronic kidney disease: a Japanese perspective on a worldwide problem. J Nephrol 2008;2I (3):305-3I2.

II. Wang Y, Chen X, Klag MJ, Caballero B. Epidemic of childhood obesity: implications for kidney disease. Adv Chronic Kidney Dis 2006; I3(4):336-35I.

12. Ritz E, Koleganova N. Obesity and chronic kidney disease. Semin Nephrol 2009;29(5):504-5I I.

13. Atkins RC. The epidemiology of chronic kidney disease. Kidney Int Suppl 2005;(94):SI4-SI8.

14. Ayodele OE, Alebiosu CO. Burden of chronic kidney disease: an international perspective. Adv Chronic Kidney Dis 2010;17(3):215-224.

15. Peter F, Evans T. Ethical dimensions of health equity. En: Evans T, Whitehead M, Diderichsen F, Bhuiya A, Wirth M, comp. Challenging inequities in health: from ethics to action. New York: Oxford University Press, 200I:25-33.

16. Macinko JA, Starfield B. Annotated Bibliography on Equity in Health, 1980-200 I. Int ] Equity Health 2002; I (I): I.

17. Diderichsen F, Evans T, Whitehead M. The social basis of disparities in health. En: Evans T, Whitehead M, Diderichsen F, Bhuiya A, Wirth M, comp. Challenging inequities in health: from ethics to action. New York: Oxford University Press, 2001:13-23.

18. Norris K, Nissenson AR. Race, gender, and socioeconomic disparities in CKD in the United States. J Am Soc Nephrol 2008; 19(7): I26I-I 270.

19. Norris KC, Agodoa LY. Unraveling the racial disparities associated with kidney disease. Kidney Int 2005;68(3):9|4-924.

20. Tareen N, Zadshir A, Martins D, Pan D, Nicholas S, Norris K. Chronic kidney disease in African American and Mexican American populations. Kidney Int Suppl 2005;(97):SI37-SI 40.

2I. Naqshbandi M, Harris SB, Esler JG, Antwi-Nsiah F. Global complication rates of type 2 diabetes in Indigenous peoples: A comprehensive review. Diabetes Res Clin Pract 2008;82(I): I- I7.

22. Jha V. Current status of chronic kidney disease care in southeast Asia. Semin Nephrol 2009;29(5):487-496.

23. Garcia-Garcia G, Renoirte-Lopez K, Marquez-Magaña I. Disparities in renal care in Jalisco, Mexico. Semin Nephrol 2010;30(I):3-7.

24. López-Cervantes M, Rojas-Russell ME, Tirado-Gómez LL, DuránArenas L, Pacheco-Domínguez RL, Venado-Estrada AA, et al. Enfermedad renal crónica y su atención mediante tratamiento sustitutivo en México. México DF: Facultad de Medicina-Universidad Nacional Autónoma de México, 2009. [Consultado 201I mayo 29]. Disponible en: http://www. dged.salud.gob.mx/opencms/opencms/contenidos/dged/descargas/ERC4may.pdf

25. Barendregt J], Van Oortmarssen G], Vos T, Murray CJ. A generic mode for the assessment of disease epidemiology: the computational basis of DisMod II. Popul Health Metr 2003; I (I):4.

26. United States Renal Data System (USRDS). 2008 Annual Data Report. [consultado 201 I mayo 29] Disponible en: http://www.usrds.org/ adr 2008.htm

27. Prakash S, O'Hare AM. Interaction of aging and chronic kidney disease. Semin Nephrol 2009;29(5):497-503. 
28. Garcia-Garcia G, Briseño-Rentería G, Luquín-Arellan VH, Gao Z, Gill J, Tonelli M. Survival among patients with kidney failure in Jalisco, Mexico. J Am Soc Nephrol 2007; I8(6): 1635-1636.

29. Horvat LD, Shariff SZ, Garg AX. Global trends in the rates of living kidney donation. Kidney Int 2009;75(I0): 1088-1098.

30. Amato D, Alvarez-Aguilar C, Castañeda-Limones R, Rodriguez E, AvilaDiaz M, Arreola F, et al. Prevalence of chronic kidney disease in an urban Mexican population. Kidney Int Suppl 2005;(97):SI I-SI7.

31. Consejo Nacional de Población. Índices de marginación 2005. [consultado 201 I mayo 29] Disponible en: http://www.conapo.gob.mx/ index.php?option $=$ com_content\&view $=$ article\&id $=|26 \&|$ temid $=204$ 32. Instituto Nacional de Salud Pública. Encuesta Nacional de Salud y Nutrición 2006 ENSANUT. [consultado 2011 mayo 29]. Disponible en: http://www.insp.mx/encuestoteca.htm

33. O'Donnell O, van Doorslaer E, Wagstaff A, Lindelow M.

Analyzing Health Equity Using Household Survey Data. A guide to techniques and their implementation. Washington: The World Bank, 2008:95-108. [consultado 20II mayo 29] Disponible en: http://web.worldbank.org/WBSITE/EXTERNAL/TOPICS/ EXTHEALTHNUTRITIONANDPOPULATION/EXTPAH/0,, contentMD K:202 I 6933 menuPK:400482 pagePK: I 48956 piPK:2 166 I 8 theSiteP $\mathrm{K}: 400476,00 . \mathrm{html}$

34. Steinke JM, Mauer M. Lessons learned from studies of the natural history of diabetic nephropathy in young type I diabetic patients. Pediatr Endocrinol Rev 2008;5 Suppl 4:958-963.

35. Rossing P. Diabetic nephropathy: worldwide epidemic and effects of current treatment on natural history. Curr Diab Rep 2006;6(6):479-483. 36. Dirks JH, de Zeeuw D, Agarwal SK, Atkins RC, Correa-Rotter R, D'Amico G, et al. Prevention of chronic kidney and vascular disease: toward global health equity--the Bellagio 2004 Declaration. Kidney Int Suppl 2005;(98):SI-SI6.

37. Foley RN, Collins AJ. The growing economic burden of diabetic kidney disease. Curr Diab Rep 2009;9(6):460-465.
38. Leoncini G, Viazzi F, Rosei EA, Ambrosioni E, Costa FV, Leonetti G, et al. Chronic kidney disease in hypertension under specialist care: the I-DEMAND study. J Hypertens 20I0;28(I):I56-I62.

39. Singh GR. Glomerulonephritis and managing the risks of chronic renal disease. Pediatr Clin North Am 2009;56(6): 1363-I382.

40. Lauder A, Schieppati A, Conte F, Remuzzi G, Batlle D. Low mortality and key aspects of delivery of care for end-stage renal disease in Italy. Scientific World Journal 2009;9:349-359.

4I. Almaguer M, Herrera R, Alfonso J, Magrans C, Manalich R, Martinez A. Primary health care strategies for the prevention of end-stage renal disease in Cuba. Kidney Int Suppl 2005;(97):S4-SIO.

42. Mazzuchi N, Schwedt E, Sola L, Gonzalez C, Ferreiro A. Risk factors and prevention of end stage renal disease in uruguay. Ren Fail 2006;28(8):617-625.

43. Schieppati A, Remuzzi G. Chronic renal diseases as a public health problem: epidemiology, social, and economic implications. Kidney Int Suppl 2005;(98):S7-SI0.

44. Gomez RA. Renal disease in Colombia. Ren Fail 2006;28(8):643-647. 45. Arogundade FA, Barsoum RS. CKD prevention in Sub-Saharan Africa: a call for governmental, nongovernmental, and community support. Am J Kidney Dis 2008;5I(3):5I5-523.

46. Khwaja A, El Kossi M, Floege J, El Nahas M. The management of CKD: a look into the future. Kidney Int 2007;72(I I): I3 |6-1323.

47. Dirks JH, Levin NW. Dialysis rationing in South Africa: a global message. Kidney Int 2006;70(6):982-984.

48. Vassalotti JA, Stevens LA, Levey AS. Testing for chronic kidney disease: a position statement from the National Kidney Foundation. Am J Kidney Dis 2007;50(2):169-180.

49. Van der Zee S, Fuster V. Enfermedades cardiovasculares y nefropatía crónica: del tratamiento al fomento de la salud. Med Clin (Barc) 2009;132 Suppl I:33-37. 